found that titrating inhaled corticosteroids using a combination of FeNO and sputum eosinophils reduced exacerbation rates and asthma symptoms compared to a conventional strategy, without additional increases in inhaled corticosteroid doses.

In conclusion, serial FeNO measurements may identify airway inflammation within close temporal proximity to changes in conventional biomarkers of eosinophilic disease such as sputum eosinophil count, with the added value of being of point-of-care. Further prospective trials are required to assess if this approach can successfully predict exacerbations or be used to titrate therapy in adults with asthma.

0 @ERSpublications

Exhaled nitric oxide home monitoring is a promising surrogate marker of eosinophilic airway inflammation in asthma http://ow.ly/108ak4

Catriona Ruth $\operatorname{Nanda}^{1}$, Amisha Singapuri ${ }^{1}$, Marcia Soares ${ }^{1}$, William Monteiro ${ }^{1}$, Salman Siddiqui ${ }^{1,2}$ and Sherif Gonem ${ }^{1,2}$ ${ }^{1}$ Institute for Lung Health, Department of Infection, Immunity and Inflammation, University of Leicester, UK.

${ }^{2}$ Both authors contributed equally.

Correspondence: Sherif Gonem, Leicester Respiratory BRU, Glenfield Hospital, Groby Road, Leicester, LE3 9QP, UK. E-mail: sg330@le.ac.uk

Received: Dec 082015 | Accepted after revision: March 042016 | First published online: April 212016

Support statement: This paper presents independent research funded by the National Institute for Health Research (NIHR). The views expressed are those of the authors and not necessarily those of the NHS, the NIHR or the Department of Health. We gratefully acknowledge the loan of NObreath devices from Bedfont Scientific Ltd. Funding information for this article has been deposited with FundRef.

Conflict of interest: Disclosures can be found alongside this article at erj.ersjournals.com

\title{
References
}

1 Green RH, Brightling CE, McKenna S, et al. Asthma exacerbations and sputum eosinophil counts: a randomised controlled trial. Lancet 2002; 360: 1715-1721.

2 Petsky HL, Cates CJ, Lasserson TJ, et al. A systematic review and meta-analysis: tailoring asthma treatment on eosinophilic markers (exhaled nitric oxide or sputum eosinophils). Thorax 2012; 67: 199-208.

3 Powell $\mathrm{H}$, Murphy VE, Taylor DR, et al. Management of asthma in pregnancy guided by measurement of fraction of exhaled nitric oxide: a double-blind, randomised controlled trial. Lancet 2011; 378: 983-990.

4 Global Strategy for Asthma Management and Prevention. Global Initiative for Asthma (GINA) 2010. www.ginasthma.org/ Date last updated: May 6, 2015. Date last accessed: December 8, 2015.

5 British Guideline on the Management of Asthma. British Thoracic Society/Scottish Intercollegiate Guidelines Network 2008. www.brit-thoracic.org.uk/guidelines/asthma-guidelines.aspx Date last updated: October 1, 2014. Date last accessed: December 8, 2015.

6 Pavord ID, Pizzichini MM, Pizzichini E, et al. The use of induced sputum to investigate airway inflammation. Thorax 1997; 52: 498-501.

7 Pizzichini MM, Popov TA, Efthimiadis A, et al. Spontaneous and induced sputum to measure indices of airway inflammation in asthma. Am J Respir Crit Care Med 1996; 154: 866-869.

8 Hashimoto S, Brinke AT, Roldaan AC, et al. Internet-based tapering of oral corticosteroids in severe asthma: a pragmatic randomised controlled trial. Thorax 2011; 66: 514-520.

9 Honkoop PJ, Loijmans RJ, Termeer EH, et al. Symptom- and fraction of exhaled nitric oxide-driven strategies for asthma control: A cluster-randomized trial in primary care. J Allergy Clin Immunol 2015; 135: 682-688.

10 Malerba M, Radaeli A, Olivini A, et al. The combined impact of exhaled nitric oxide and sputum eosinophils monitoring in asthma treatment: a prospective cohort study. Curr Pharm Des 2015; 21: 4752-4762.

\section{Quality standards for the management of bronchiectasis in Italy: a national audit}

\section{To the Editor:}

Although historically considered a neglected disease, bronchiectasis has become a disease of renewed interest over recent decades in light of an increase in prevalence and a substantial burden on healthcare systems [1-3]. In 2010, the British Thoracic Society (BTS) published guidelines on the management of bronchiectasis in adults, along with specific quality standards $[4,5]$. To date, these represent the only 
quality standards available in Europe. These have been tested over a number of years in the UK with progressive improvements in the standard of care [6]. No national guidelines are available in Italy and no indications on which guideline should be followed have been given by the Italian Society of Respiratory Medicine (SIP). There are limited published data on the quality of bronchiectasis care in Europe outside of the UK. The BTS standards have not been tested in continental Europe or in Italy, where information on characteristics and management of bronchiectasis patients are lacking.

A national audit was conducted by the SIP on adult patients with bronchiectasis who attended secondary care clinics in 32 hospitals across Italy in 2014 and 2015. An invitation to participate in the audit was sent to the chiefs of pulmonary departments in Italy, as well as members of the SIP. They were asked to complete an electronic case report form (CRF) for each patient enrolled, concordant with the CRF used in the 2012 UK audit [6]. A total of 1361 records were submitted from 32 institutions across the country. The majority of the patients were female $(n=719,53 \%)$ and the median (interquartile range (IQR)) age was 70 (59-77) years. Pseudomonas aeruginosa was isolated at least once during the previous year in $20 \%$ of the patients, enteric Gram-negative organisms in $7.8 \%$ and methicillin-resistant Staphylococcus aureus (MRSA) in $2.9 \%$. A total of $158(12 \%)$ patients had a chronic infection with P. aeruginosa, defined as the presence of this pathogen in at least two consecutive sputum samples collected in stable state over 1-year period [7].

$43 \%$ of the patients had two or more exacerbations per year, while $18 \%$ had three or more exacerbations per year, with a median (IQR) of 2 (2-3) exacerbations per year. $49 \%$ had been hospitalised at least once in the previous year for lower respiratory tract infections (LRTI). The median (IQR) number of antibiotic courses for LRTI in the previous year was $2(2-3)$, with $40 \%$ of the study population receiving three or more antibiotic courses in the previous year. Specifically, $36 \%$ of them received at least one course of intravenous antibiotics because of an exacerbation during the previous year.

The prevalence of chronic infection with $P$. aeruginosa was lower than the prevalence of patients with two to three or more exacerbations or hospitalisations per year, and some explanations might be suggested for that. On one hand, we can speculate that frequent exacerbators are also those with either an intermittent infection with $P$. aeruginosa or chronic infections with other bacteria, as recently reported [8], and that microbiology is just one of several bronchiectasis features responsible for bad outcomes [7]. On the other hand, hospitalisations for LRTI in bronchiectasis patients might be also due to factors not strictly related to bronchiectasis and infection, such as the presence of decompensated comorbidities or failure of oral antibiotic therapy [9]. The rate of $P$. aeruginosa isolation (20\%) in the present audit is very similar to the reported prevalence of $P$. aeruginosa in 21 cohorts recently included in a meta-analysis where the mean frequency of isolation was $21.4 \%$ [10]. We suspect that the frequency of $P$. aeruginosa may be underestimated due to a lack of regular sputum sampling in Italy (see later).

A total of 119 (9\%) patients were on long-term antibiotic treatment, either orally or by inhalation. 7.8\% had received long-term oral antibiotics for $>28$ days and among them, 74 were taking azithromycin, 11 levofloxacin, nine ciprofloxacin, three moxifloxacin, three trimethoprim/sulfamethoxazole, two clarithromycin and one amoxicillin/clavulanate. Only $1.1 \%$ of the patients had received long-term inhaled/ nebulised antibiotics for $>28$ days. Among them, 10 were on tobramycin, three on colomycin and two on gentamicin. Four patients were on both oral and inhaled/nebulised long-term antibiotic treatment.

The BTS quality standard for the management of patients with bronchiectasis evaluated in the present audit comprises 10 statements. The adherence to these standards is depicted in figure 1.

In order to make a diagnosis of bronchiectasis, the first quality statement recommends a computed tomography (CT) of the chest using 1-mm slices. In this SIP audit, 93\% of the patients had a CT scan, although only $46 \%$ had a high-resolution CT scan. Bronchiectasis was diagnosed by chest radiography in $5 \%$ and by bronchography in $1 \%$ of the rest of the population, while $1 \%$ had a clinical diagnosis alone. The second statement recommends that patients should be investigated for allergic bronchopulmonary aspergillosis (ABPA), common variable immunodeficiency (CVID) and cystic fibrosis (CF), the last of these if indicated, as these are specific and treatable causes of bronchiectasis [11]. In the SIP audit, only 435 (32\%) patients were tested for at least one of the above: $17 \%$ of the patients had been investigated for ABPA, $22 \%$ for CVID and $5.5 \%$ for CF. Furthermore, 9.3\% of patients were tested for IgG subclasses, $4.7 \%$ for HIV, $8.2 \%$ for $\alpha_{1}$-antitripsin deficiency, $18 \%$ for autoantibodies and $2.6 \%$ for either saccharin test or electronic microscopy for ciliary dysfunction. Finally, no tests to investigate the aetiology of bronchiectasis were performed in 59\% of the patients. The third and fourth statements require that people with bronchiectasis have sputum bacterial culture when clinically stable recorded at least once each year and at the start of an exacerbation before initiating antibiotics. $27 \%$ had at least one sputum sent for bacterial culture in stable state during the previous year and almost $50 \%$ at the beginning of an exacerbation. The fifth statement proposes that people with bronchiectasis are taught appropriate airway clearance techniques by a specialist respiratory physiotherapist 


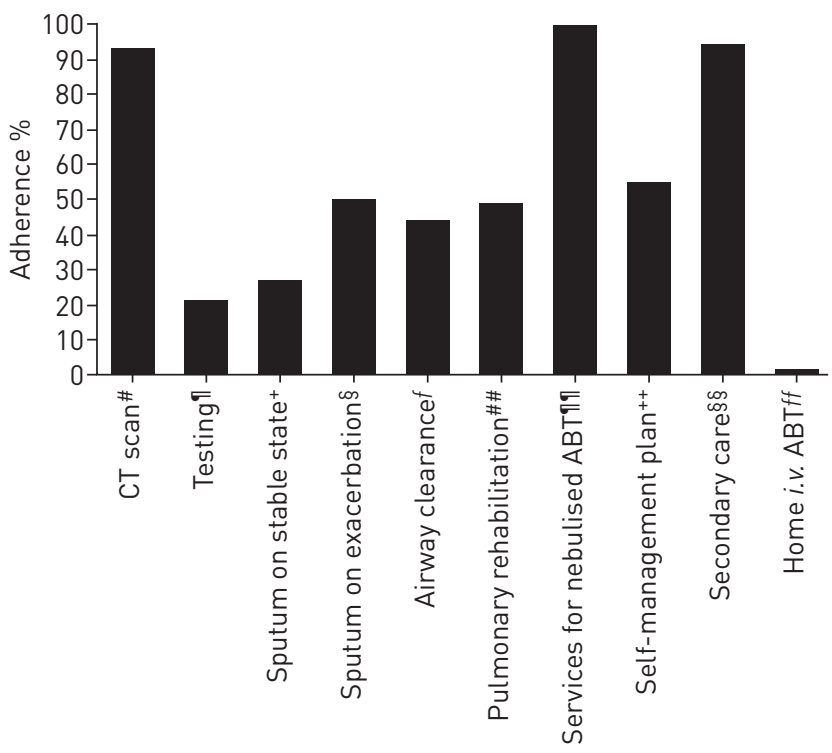

FIGURE 1 Summary of the quality statements for bronchiectasis in adults and percentage adherence in the Italian Society of Respiratory Medicine (SIP) Audit. \# : people with a clinical diagnosis of bronchiectasis have the diagnosis confirmed by chest computed tomography (CT) scan (using $1 \mathrm{~mm}$ slices). " : people with bronchiectasis to be investigated for allergic bronchopulmonary aspergillosis, common variable immunodeficiency and cystic fibrosis (the last of these, if indicated), as these are specific treatable causes. ${ }^{+}$: people with bronchiectasis have sputum bacteriology culture when clinically stable recorded at least once each year. ${ }^{\S}$ : sputum is sent for bacterial culture at the start of an exacerbation before starting antibiotics; empirical antibiotic treatment to start as soon as feasible and not await the sputum culture results. ${ }^{f}$ : people with bronchiectasis are taught appropriate airway clearance techniques by a specialist respiratory physiotherapist and advised of the frequency and duration with which these should be carried out. \#\# : people with bronchiectasis to attend pulmonary rehabilitation if they have breathlessness affecting their activities of daily living. "ा?: services for people with bronchiectasis to include provision of nebulised prophylactic antibiotic therapy (ABT) for suitable patients supervised by a respiratory specialist. ${ }^{++}$: people with bronchiectasis to have an individualised, written self-management plan. ${ }^{\S}$ : people with bronchiectasis who meet the criteria for continuing secondary care to be managed by a multidisciplinary team led by a respiratory physician. ${ }^{f f}$ : services for people with bronchiectasis to include provision of home intravenous ABT for exacerbations in selected patients.

and advised of the frequency and duration with which these should be carried out. In the SIP audit, only $44 \%$ of the patients were taught chest clearance techniques by a specialist respiratory physiotherapist. The sixth statement proposes that people with bronchiectasis and breathlessness affecting their activities of daily living should attend pulmonary rehabilitation, in order to improve their exercise capacity and health status. $49 \%$ of Italian patients had breathlessness and among those, $49 \%$ attended a pulmonary rehabilitation programme. The seventh statement requires that there should be provision of nebulised prophylactic antibiotics for suitable patients supervised by a respiratory specialist. All 20 patients receiving long-term nebulised antibiotics for $>1$ month were supervised by respiratory physicians using off-label treatment. The eighth statement is that people with bronchiectasis should have an individualised written self-management plan in order to manage their condition and to recognise, respond to and reduce the occurrence of exacerbations. In the SIP audit, 56\% of the patients shared an individualised written self-management plan. The ninth standard is that people with bronchiectasis who meet the criteria for continuing secondary care should be managed by a multidisciplinary team led by a respiratory physician. These are patients with chronic P. aeruginosa, mycobacteria or MRSA, three or more exacerbations per year, receiving long-term antibiotic treatment, bronchiectasis associated with rheumatoid arthritis, immune deficiency, inflammatory bowel disease, primary ciliary dyskinesia or ABPA, and advanced disease, and/or considering lung transplantation. Among the 665 patients who met these criteria, the majority (94\%) was seen by respiratory physicians at a secondary care level $(20 \%$ were seen every 2 months, $32 \%$ every 4 months, $36 \%$ were seen 6 -monthly and $12 \%$ once a year) and $6 \%$ by general practitioners. Furthermore, only four out of the 32 study centres participating in the audit have a specific bronchiectasis clinic taking care of 221 (16\%) patients in total. The development of tertiary-care bronchiectasis clinics should be a priority at national level in order to offer the most severe patients a better and multidisciplinary management of their disease. The final statement concerns services for people with bronchiectasis, which should include provision of home i.v. antibiotic treatment for exacerbations in selected patients. In the audit, only $2.3 \%$ were offered domiciliary i.v. antibiotics. Only one out of 32 centres was able to offer a domiciliary treatment with i.v. antibiotics for an exacerbation. 
The majority of the BTS recommendations for the management of bronchiectasis in adults were not met in Italy, with six out of 10 being reached in $<50 \%$ of the patients. A lower percentage of patients undergoing high-resolution CT scan and standard testing for bronchiectasis aetiology is reported in the present SIP audit in comparison to the 2012 UK audit [6]. Five more areas require particular attention, including: monitoring sputum bacteriology; promoting airway clearance taught by a specialist respiratory physiotherapist and pulmonary rehabilitation in selected patients; and developing services to allow domiciliary intravenous antibiotic treatment [12]. Possible reasons for low adherence to the quality standards include the absence of Italian and European guidelines, and lack of awareness of the disease and of the evidence base to support recommendations. Following the results of this audit, we suggest, on one hand, increasing educational activities on bronchiectasis at a national level according to the Harmonising Education in Respiratory Medicine for European Specialists (HERMES) curriculum and promoting access to the HERMES diploma, and on the other hand, to develop and subsequently implement national or European guidelines. Results of this intervention might lead to a better care of our patients, as the third UK audit demonstrated in comparison to the previous ones [6]. One tool to monitor the implementation of standard operating procedures in Italy will be the SIP national registry of adult patients with bronchiectasis that has been recently developed and linked to the EMBARC European registry [13].

0 @ERSpublications

The majority of the quality standards for the management of bronchiectasis in adults are not met in Italy http://ow.ly/YKMpU

Stefano Aliberti ${ }^{1}$, Adam T. Hill ${ }^{2}$, Marco Mantero ${ }^{3}$, Salvatore Battaglia $\oplus^{4}$, Stefano Centanni ${ }^{5}$, Salvatore Lo Cicero ${ }^{6}$, Donato Lacedonia $^{7}$, Marina Saetta ${ }^{8}$, James D. Chalmers ${ }^{9}$ and Francesco Blasi ${ }^{3}$ on behalf of the SIP Bronchiectasis Audit Working Group ${ }^{10}$

${ }^{1}$ School of Medicine and Surgery, University of Milan Bicocca, AO San Gerardo, Monza, Italy. ${ }^{2}$ Dept of Respiratory Medicine, Royal Infirmary and University of Edinburgh, Edinburgh, UK. ${ }^{3}$ Dept of Pathophysiology and Transplantation, Università degli Studi di Milano, IRCCS Fondazione Ca' Granda, Ospedale Maggiore Policlinico, Milan, Italy. ${ }^{4}$ Dipartimento Biomedico di Medicina Interna e Specialistica (DiBiMIS), Sezione di Malattie Cardio-Respiratorie ed Endocrino-Metaboliche, University of Palermo, Palermo, Italy. ${ }^{5}$ Respiratory Unit, San Paolo Hospital, Dipartimento Scienze della Salute, Università degli Studi di Milano, Milan, Italy. ${ }^{6}$ SC Pneumologia, Grande Ospedale Metropolitano Niguarda, Milan, Italy. ${ }^{7}$ Dipartimento di Scienze Mediche e Chirurgiche, Università di Foggia, Foggia, Italy. ${ }^{8}$ Unità Operativa Complessa di Pneumologia, Dipartimento di Scienze Cardiologiche, Toraciche e Vascolari, Azienda Ospedaliera e Università degli Studi di Padova, Padua, Italy. ${ }^{9}$ College of Medicine, University of Dundee, Dundee, UK.

${ }^{10} \mathrm{~A}$ list of the SIP Bronchiectasis Audit Working Group investigators can be found in the Acknowledgements section.

Correspondence: Stefano Aliberti, School of Medicine and Surgery, University of Milan Bicocca, Respiratory Unit, AO San Gerardo, Via Pergolesi 33, 20052, Monza, Italy. E-mail: stefano.aliberti@unimib.it

Received: Jan 302016 | Accepted after revision: Feb 172016 | First published online: April 132016

Support statement: This study was supported by SIP (Società Italiana di Pneumologia) and the European Multicentre Bronchiectasis Audit and Research Collaboration (EMBARC) (www.bronchiectasis.eu). EMBARC is a European Respiratory Society Clinical Research Collaboration and has received funding from the European Respiratory Society, Bayer HealthCare and the Aradigm Corporation. James D. Chalmers acknowledges fellowship support from the UK Medical Research Council and the Wellcome Trust. Funding information for this article has been deposited with FundRef.

Conflict of interest: Disclosures can be found alongside this article at erj.ersjournals.com

Acknowledgements: The SIP Bronchiectasis Audit Working Group investigators are Carmela Morrone (Dept of Pathophysiology and Transplantation, University of Milan, IRCCS Fondazione Ca' Granda, Ospedale Maggiore Policlinico, Milan, Italy), Marco Basile (Di.Bi.MIS, Sezione di Malattie Cardio-Respiratorie ed Endocrino-Metaboliche, University of Palermo, Palermo, Italy), Giuseppe Francesco Sferrazza Papa (Dept of Health Sciences, University of Milan, ASST Santi Paolo e Carlo, Milan, Italy), Caterina Conti (SC Pneumologia, Grande Ospedale Metropolitano Niguarda, Milan, Italy), Maria Pia Foschino Barbaro (Dipartimento di Scienze Mediche e Chirurgiche, Università di Foggia, Foggia, Italy), Kim Lokar-Oliani (Unità Operativa Complessa di Pneumologia, Dipartimento di Scienze Cardiologiche, Toraciche e Vascolari, Azienda Ospedaliera e Università degli Studi di Padova, Padua, Italy), Pietro Schino (UOS Fisiopatologia Respiratoria, Ente ecclesiastico Ospedale Generale Regionale "F. Miulli", Acquaviva delle Fonti, Bari, Italy), Michele Vitacca (Fondazione "S. Maugeri" IRCCS, Pneumologia Riabilitativa, Lumezzane, Brescia, Italy), Francesco Menzella (SC Pneumologia, Arcispedale S. Maria Nuova, IRCCS, Reggio Emilia, Italy), Alessandro Sanduzzi (Università degli Studi di Napoli Federico II, Dipartimento di Pneumologia ed Oncologia, Ospedale Monaldi, UOC di Pneuotisiologia, Naples, Italy), Pierfranco Usai (SC Pneumologia Ospedale di Vimercate, Vimercate, Italy), Mauro Carone (Fondazione "S. Maugeri' IRCCS, Centro Medico di Cassano Murge, Divisione di Pneumologia, Bari, Italy), Daniela Rita Bonardi (UOC Pneumologia Riabilitativa INRCA IRCCS Casatenovo, Lecco, Italy), Nunzio Crimi (UO di Pneumologia Riabilitativa, AOU "Policlinico Vittorio Emanuele" di Catania, Catania, Italy), Gianfranco Schiraldi (IRCSS Auxologico Italiano, Milan, Italy), Angelo Corsico (SC Pneumologia, Fondazione IRCCS Policlinico San Matteo, Dipartimento di Medicina Interna e Terapia Medica, Università di Pavia, Pavia, Italy), Mario Malerba (Dipartimento di Medicina Interna, Spedali Civili ASST e Università di Brescia, Brescia, Italy), Francesca Becciu (SC Pneumologia Riabilitativa-UTIR, PO Sondalo, ASST Valtellina Alto Lario, Sondalo, Italy), Pierachille Santus (Università degli Studi di Milano, Pneumologia Riabilitativa Fondazione Salvatore Mugeri Istituto 
Scientifico di Milano, IRCCS, Milan, Italy), Giuseppe Girbino (Malattie Apparato Respiratorio, Università di Messina, UOC di Pneumologia, Policlinico Universitario, Messina, Italy), Antonio Foresi (ASST Nord Milano, UOC Pneumologia, PO di Sesto San Giovanni, Sesto San Giovanni, Italy), Vittoria Comellini (Dipartimento DIMES, Alma Mater Bologna, Pneumologia e Terapia Intensiva Respiratoria, Ospedale Sant'Orsola Malpighi, Bologna, Italy), Claudia Stochino (Pneumotisiologia, ASST Valtellina e Alto Lario, Sondalo, Italy), Piero Ceriana (Pneumologia Riabilitativa, IRCCS Fondazione Maugeri, Pavia, Italy), Valentina Conti (Modulo Funzionale di Pneumologia, Istituto Sicurezza Sociale, Ospedale di Stato, San Marino), Fulvio Braido (Clinica Malattie Respiratorie e Allergologia, Azienda Ospedaliera Universitaria IRCCS San Martino di Genova, Genoa, Italy), Vincenzo Mastrosimone (UO Pneumologia e Riabilitazione, Fondazione Salvatore Mugeri Istituto Scientifico di Marina di Ginosa, Taranto, Italy), Nicola Ciancio (AUO Policlinico Vittorio Emanuele, UO Pneumologia, Catania, Italy), Marialma Berlendis (UO Pneumologia, AO Spedali Civili di Brescia, Brescia, Italy), Francesco De Blasio (Unità Funzionale di Pneumologia e Riabilitazione Respiratoria, Casa di Cura Clinic Center SpA, Naples, Italy), Sergio Celestino Conte (UOC Pneumologia, Presidio Ospedaliero Vittorio Veneto, ULSS7 Regione Veneto, Treviso, Italy) and Alessandro Vatrella (Cattedra di Malattie Respiratorie, Università di Salerno, Salerno, Italy).

The authors would like to acknowledge the assistance of C. Recalcati (SIP Service), M. Basile and E. Mazzuca (University of Palermo, Palermo, Italy), F. Alfano (University of Milan, ASST Santi Paolo e Carlo, Milan, Italy), M. Chiericozzi (Grande Ospedale Metropolitano Niguarda, Milan, Italy), G. Patricelli (Università di Foggia, Foggia, Italy), P. Peditto (Azienda Ospedaliera e Università degli Studi di Padova, Padua, Italy), L. Bianchi (Fondazione "S. Maugeri" IRCCS, Lumezzane, Brescia, Italy), C. Galeone and S. Taddei (Arcispedale S. Maria Nuova, IRCCS, Reggio Emilia, Italy), M. Accardo and M. Mosella (Università degli Studi di Napoli Federico II, Ospedale Monaldi, Naples, Italy), W. Casali (Ospedale di Vimercate, Vimercate, Italy), M. Aliani and V. Turchiarelli (Fondazione "S. Maugeri" IRCCS, Centro Medico di Cassano Murge, Bari, Italy), E. Guffanti (INRCA IRCCS Casatenovo, Lecco, Italy), S. Ferri and R. Campisi (AOU "Policlinico - Vittorio Emanuele" di Catania, Catania, Italy), V. Conio and L. Saracino (Fondazione IRCCS Policlinico San Matteo, Università di Pavia, Pavia, Italy), A. Radaeli (Spedali Civili ASST e Università di Brescia, Brescia, Italy), A. Papalia (ASST Valtellina Alto Lario, Sondalo, Italy), R. Raccanelli and F. Giovannelli (Università degli Studi di Milano, Fondazione Salvatore Mugeri Istituto Scientifico di Milano - IRCCS, Milan, Italy), S. Calcaterra (Università di Messina, Policlinico Universitario, Messina, Italy), A. Pelucchi (ASST Nord Milano, Sesto San Giovanni, Italy), S. Nava (Alma Mater Bologna, Ospedale Sant'Orsola Malpighi, Bologna, Italy), E. Rossi and S. Ruli (Istituto Sicurezza Sociale, Ospedale di Stato, San Marino), S. Garuti and M. Ferrari (Azienda Ospedaliera Universitaria IRCCS San Martino di Genova, Genoa, Italy), G. Di Maria and D. Lombardo (AUO Policlinico Vittorio Emanuele, Catania, Italy), G. Steinhilber (AO Spedali Civili di Brescia, Brescia), M. De Martino (Casa di Cura Clinic Center SpA, Naples, Italy), and C. Vitale (Università di Salerno, Salerno, Italy).

\section{References}

1 Ringshausen FC, de Roux A, Diel R, et al. Bronchiectasis in Germany: a population-based estimation of disease prevalence. Eur Respir J 2015; 46: 1805-1807.

2 Quint JK, Millett ER, Joshi M, et al. Changes in the incidence, prevalence and mortality of bronchiectasis in the UK from 2004 to 2013: a population-based cohort study. Eur Respir J 2016; 47: 186-193.

3 Chalmers JD, Aliberti S, Blasi F. Management of bronchiectasis in adults. Eur Respir J 2015; 45: 1446-1462.

4 Pasteur MC, Bilton D, Hill AT et al. British Thoracic Society guideline for non-CF bronchiectasis. Thorax 2010; 65: Suppl. 1, i1-i58.

5 Hill AT, Bilton D, Brown J. BTS Quality Standards for clinically significant bronchiectasis in adults. Br Thorac Soc Rep 2012; 4: 1-16.

6 Hill AT, Routh C, Welham S. National BTS bronchiectasis audit 2012: is the quality standard being adhered to in adult secondary care? Thorax 2014; 69: 292-294.

7 Chalmers JD, Goeminne P, Aliberti S, et al. The bronchiectasis severity index. An international derivation and validation study. Am J Respir Crit Care Med 2014; 189: 576-585.

8 Aliberti S, Lonni S, Dore S, et al. Clinical phenotypes in adult patients with bronchiectasis. Eur Respir J 2016; 47: $1113-1122$.

9 Aliberti S, Ramirez J, Cosentini R, et al. Low CURB-65 is of limited value in deciding discharge of patients with community-acquired pneumonia. Respir Med 2011; 105: 1732-1738.

10 Finch S, McDonnell MJ, Abo-Leyah H, et al. A comprehensive analysis of the impact of Pseudomonas aeruginosa colonization on prognosis in adult bronchiectasis. Ann Am Thorac Soc 2015; 12: 1602-1611.

11 Lonni S, Chalmers JD, Goeminne PC, et al. Etiology of non-cystic fibrosis bronchiectasis in adults and its correlation to disease severity. Ann Am Thorac Soc 2015; 12: 1764-1770.

12 Bedi P, Sidhu MK, Donaldson LS, et al. A prospective cohort study of the use of domiciliary intravenous antibiotics in bronchiectasis. NPJ Prim Care Respir Med 2014; 24: 14090.

13 Chalmers JD, Aliberti S, Polverino E, et al. The EMBARC European Bronchiectasis Registry: protocol for an international observational study. ERJ Open Res 2015; 1: 00081-2015. 